\title{
PRIMER REGISTRO DE XIPHACTINUS LEIDY, 1870 (TELEOSTEI, ICHTHYODECTIFORMES) EN EL CRETÁCICO SUPERIOR DE AMÉRICA DEL SUR (FORMACIÓN LA LUNA, VENEZUELA)
}

\author{
JORGE CARRILLO-BRICEÑO \\ Alcaldía Bolivariana de Urumaco, Museo Paleontológico, Urumaco, Venezuela.jorgedcb100@gmail.com \\ JESÚS ALVARADO-ORTEGA \\ Instituto de Geología, Universidad Nacional Autónoma de México, Circuito de la Investigación s/n, Ciudad Universitaria, \\ Delegación Coyoacán, D.F., 04510, México.alvarado@geologia.unam.mx \\ CARLOS TORRES \\ Departamento de Geología, Complejo Cementero Cemento Andino, Monay, Trujillo, Venezuela.kaly_7@hotmail.com
}

\begin{abstract}
THE FIRST RECORD OF XIPHACTINUS LEIDY, 1870 (TELEOSTEI, ICHTHYODECTIFORMES) IN THE UPPER CRETACEOUS OF SOUTH AMERICA (LA LUNA FORMATION, VENEZUELA). This paper describes the fossil remains of an Ichthyodectiform fish collected in the Cenomanian limestones belonging to La Aguada Member, La Luna Formation, near Monay, Candelaria Municipality, Trujillo State, western Venezuela. This fossil consists of fragments of the nasal region of the skull, both jaws, and four vertebrae of the same individual. Despite the fragmentary condition of this fossil, its preserved characteristics show that it is a representative of the genus Xiphactinus Leidy. These characters are the occurrence of conic, caniniform teeth of irregular size set within deep alveolar cavities forming a single row in the maxilla, premaxilla, and dentary bones; hypertrophied premaxilla teeth; and a wide maxilla-premaxilla articulation that involves the entire height of both bones. Unfortunately, there are not enough characters preserved to determinate the specific identity of this fossil; nevertheless, this finding of Xiphactinus in a South American locality represents the first report of this genus from the marine domains of the Mesozoic Gondwana. This report extends the paleobiogeographic distribution of Xiphactinus that is well known through previous findings in Cretaceous (Albian-Maastrichthian) marine localities, within the Inland Sea of North America and the northern border of the Tethys Sea in Europe.
\end{abstract}

Key words: Ichthyodectiformes, Xiphactinus, Cretaceous, La Luna Formation, Venezuela.

RESUMO - O presente trabalho descreve restos de um peixe Ichthyodectiforme coletado nos calcários cenomanianos do Membro La Aguada, Formación La Luna, nas proximidades de Monay, Municipio Candelaria, Estado de Trujillo, Venezuela. O material é composto por restos cranianos da região nasal, mandíbulas e quatro vértebras pertencentes a um mesmo exemplar. Apesar do material apresentar-se bastante fragmentário, suas características permitem reconhecer um representante do gênero Xiphactinus Leidy. Estas características incluem a presença de dentes caniniformes, cônicos, de tamanho irregular, que ocupam alvéolos profundos e formam uma fila na maxila, premaxila e dentário; dentes premaxilares hipertrofiados; assim como uma ampla articulação maxilar-premaxilar que envolve toda a altura de ambos ossos. Infelizmente, não há caracteres suficientes para determinação do exemplar a nível específico; no entanto, o achado de Xiphactinus na América do Sul representa seu primeiro registro dentro do domínio marinho mesozoico do Gondwana. Este registro amplia a distribuição paleobiogeográfica do género, previamente bem conhecido nos afloramentos marinhos do Cretáceo (Albiano-Maastrichtiano), em localidades do Mar Interior da América do Norte e da borda septentrional do Mar de Tétis na Europa.

Palavras-chave: Ichthyodectiformes, Xiphactinus, Cretáceo, Formação La Luna, Venezuela.

\section{INTRODUCCION}

Los Ichthyodectiformes constituyen un grupo extinto de peces teleósteos basales que vivieron desde el Jurásico Medio hasta el final del Cretácico. Aunque el primer miembro de este grupo fue descrito como un reptil a principios del siglo XIX, Saurocephalus Harlan, 1824; fueron necesarios más de cien años para que Bardack \& Sprinkle (1969) reconocieran que este pez y sus formas afines forman parte de un grupo natural, el orden Ichthyodectiformes. Estos peces han sido recolectados en rocas formadas en depósitos marinos de Europa, América del Norte, América del Sur, Antártica, África, Medio Oriente, Asia y Australia (e.g., Bardack, 1965; Schaeffer \& Patterson, 1984; Tanimoto \& Kinkyo, 2001; Arratia et al., 2004).

Los Ichthyodectiformes hasta ahora conocidos suman 
25 géneros, donde se incluyen cerca de 60 especies nominales (e.g. Taverne, 2010). Durante el Jurásico, los ichthyodectiformes estuvieron representados tan sólo por cuatro géneros con especies relativamente pequeñas, que alcanzaron el medio metro de longitud y que aparentemente se extinguieron en este mismo periodo. En contraste, sus representantes cretácicos son mucho más numerosos (21 géneros) y de tallas mayores; y entre ellos Xiphactinus Leidy, 1870 , es sin duda el más famoso debido al aspecto feroz de sus poderosas mandíbulas, armadas con enormes dientes, por contar con ejemplares donde se conserva el cuerpo completo de sus presas, y porque el registro de ejemplares de hasta 6 metros de longitud hacen de Xiphactinus el pez teleósteo más grande hasta ahora conocido (Everhart et al., 2010).

Recientemente, fragmentos de la cabeza y columna vertebral pertenecientes a un ejemplar del género Xiphactinus fueron descubiertos en rocas calizas cenomanianas explotadas en la cantera de la compañía "Cementos Andinos", ubicada entre las coordenadas $09^{\circ} 37^{\prime} \mathrm{N}$ y $70^{\circ} 24^{\prime} \mathrm{O}$, al este del Lago de Maracaibo, a $17 \mathrm{~km}$ al noreste del poblado de Monay, dentro de los límites del Municipio Candelaria, Estado Trujillo, en los Andes de Venezuela (Figura 1). Actualmente, este fósil está depositado en el Museo Tulene Bertoni, en la ciudad de Valera, Estado de Trujillo, Venezuela. El objetivo de este artículo es proporcionar una descripción detallada de este fósil, demostrar su pertenencia a Xiphactinus y discutir las implicaciones que este hallazgo tiene para dicho género.

Las rocas extraídas de la cantera "Cementos Andinos" pertenecen a la Formación La Luna. Esta unidad litoestratigráfica es una secuencia de rocas marinas depositadas a lo largo del margen pasivo del Norte de América del Sur, durante el Ceomaniano-Campaniano y dentro un ambiente somero bajo condiciones anóxico-disóxicas (Zapata et al., 2003). Esta formación es la fuente más importante de hidrocarburos en ambos lados de la frontera entre Venezuela y Colombia. Antes de ser formalmente nombrada por Garner (1926), como una unidad compuesta por la alternancia de estratos laminados de calizas bituminosas y lutitas calcáreas obscuras portadoras de concreciones discoidales, la Formación La Luna fue reconocida como "La Luna Limestones" y "Quebrada La Luna" (Hedberg \& Sass, 1937, p. 79). Inicialmente Renz (1959), basado en las secuencias sedimentarias de esta formación expuestas en la región de los estados Trujillo y Lara, propuso que la Formación La Luna consta de tres miembros:

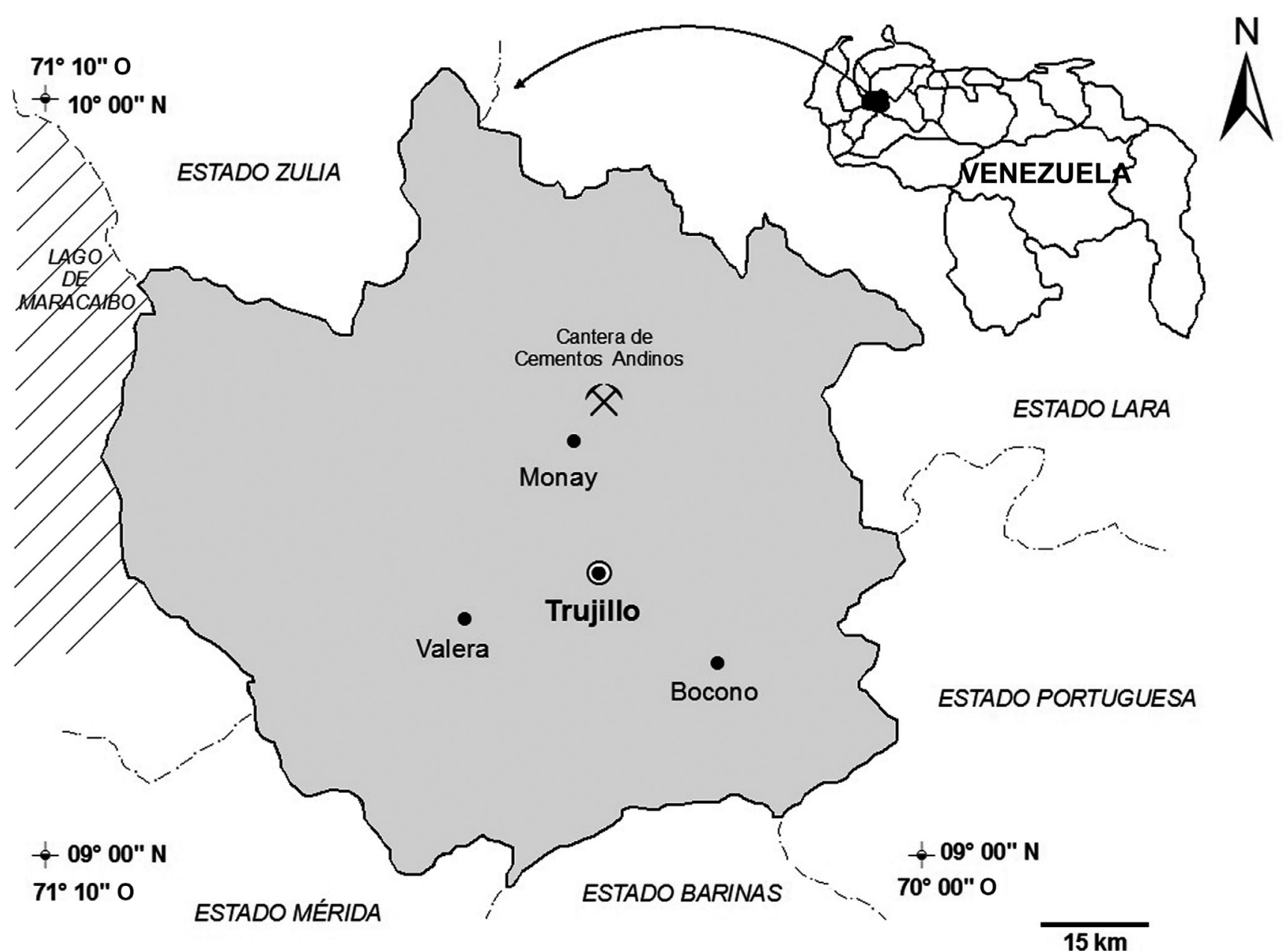

Figura 1. Ubicación de la Cantera de Cementos Andinos, en las cercanías de Monay, Estado Trujillo, Venezuela, donde fue colectado el ejemplar MT-G-070.

Figure 1. Location of Cementos Andinos Quarry, near Monay, Trujillo State, where the specimen MT-G-070 was collected. 
La Aguada, Chejendé y Timbetes (Figura 2); sin embargo, más tarde se reconoció las fosforitas de Tres Esquinas, las cuales son el miembro más joven de esta unidad geológica (de Romero et al., 2003). Aunque la edad CenomanianoSantoniano de esta formación ha sido determinada a partir de estudios bioestratigráficos (e.g. Renz, 1959; Lorente et al., 1996) y por estratigrafía isotópica (Davis, 1997), de acuerdo con de Romero et al. (2003, p. 362-363) su depósito no fue homogéneo. Por un lado, el Miembro La Aguada comenzó a ser depositado entre el Albiano tardío y el Cenomaniano medio en el área de San Felipe (región de Trujillo y Lara), e inició en el Cenomaniano medio en la región de la Sierra de Perijá, desde donde progresivamente se extendió hacia el sur hasta las regiones de Táchira y Mérida. Por otro lado, el depósito del Miembro Tres Esquinas que representa el episodio final de esta formación culminó cerca del límite superior del Santoniano en la región de Trujillo y Lara, durante el Campaniano medio en la región de Mérida, y hacia el Campaniano tardío en las regiones de Táchira y Perijá.

La litología presente en la cantera "Cementos Andinos", donde se recolectó el ejemplar motivo de este artículo, está compuesta por una secuencia de calizas y lutitas calcáreas fosilíferas de color entre gris oscuro y negro, bien laminadas, fétidas y ricas en materia orgánica. Estas rocas son portadoras de abundantes concreciones calcáreas elipsoidales y discoidales, que son características del Miembro La Aguada. La edad Cenomaniana del miembro La Aguada en esta región de Venezuela es reconocida a partir de sus foraminíferos y ammonites (Renz, 1959).

\section{MATERIAL Y MÉTODOS}

El material fósil fue encontrado dentro de un fragmento de caliza de alta dureza. La preparación de este ejemplar se llevó a cabo mediante la disolución de la matriz calcárea a través de la aplicación soluciones acuosas de ácido acético al $5 \%$. Ajugas de disección y excavadores odontológicos fueron empleados para remover mecánicamente algunos pequeños restos de la matriz rocosa de la superficie del fósil.

El material de comparación empleado en este trabajo comprende los siguientes ejemplares: Xiphactinus audax, AMNH 7350, premaxilar y maxilar casi completo (holotipo de Portheus molossus); AMNH 1673, cabeza y primeras vértebras abdominales; FHSM VP 333, ejemplar completo y articulado; FHSM VP 2973, cráneo desarticulado; FHSM VP 2175; dentario desarticulado; Ichthyodectes (anades) ctenodon, AMNH 2005, cabeza y parte anterior de la región abdominal.

Abreviaturas institucionales. AMNH, American Museum of Natural History, New York, Estados Unidos de Norteamérica; FHSM VP, Sternberg Museum of Natural History, Kansas, Estados Unidos de Norteamérica; MT-G, Museo Tulene Bertoni, ciudad de Valera, Estado de Trujillo, Venezuela.

Abreviaturas anatómicas. La nomenclatura y abreviaciones anatómicas empleada en este trabajo son retomadas de vários autores (e.g. Bardack \& Sprinkle, 1965, Patterson \& Rosen, 1977).

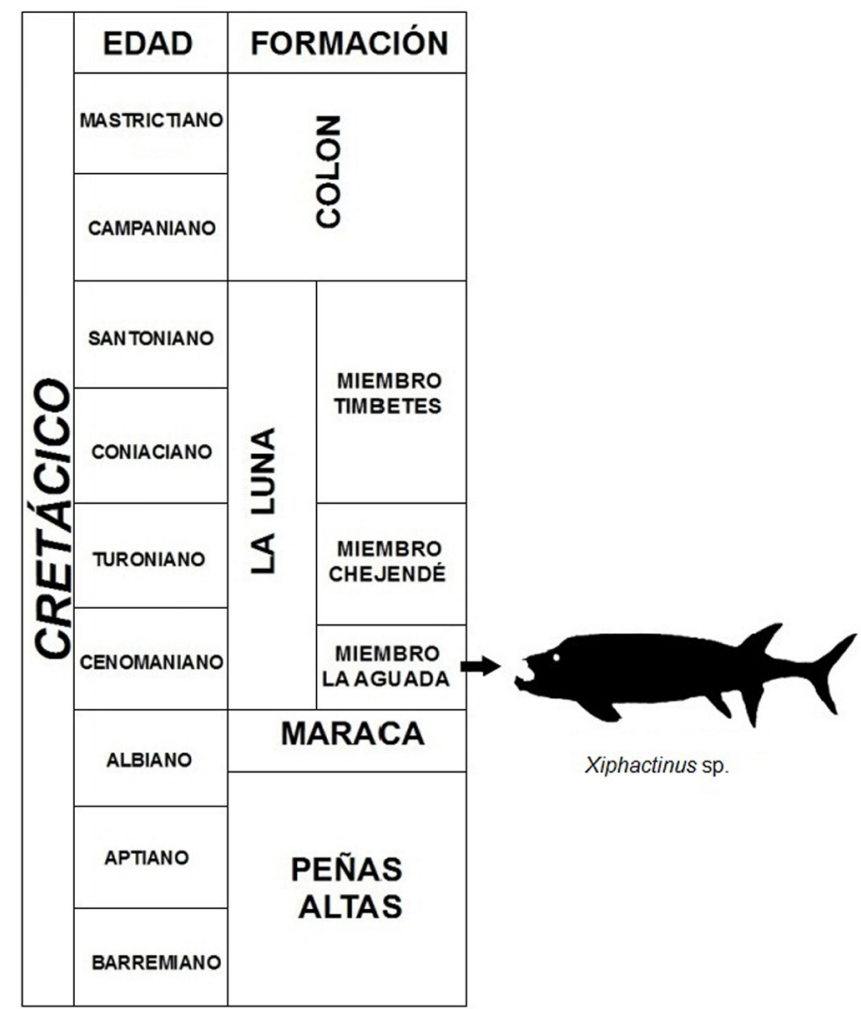

Figura 2. Unidades litoestratigráficas del Cretácico de la región de Chejendé, en las cercanías de Monay, Estado Trujillo (modificado de Renz, 1959).

Figure 2. Cretaceous lithostratigraphic units of the Chejendé region, near Monay, Trujillo State (modified from Renz, 1959).

\section{PALEONTOLOGÍA SISTEMÁTICA}

Orden ICHTHYODECTIFORMES Bardack \& Sprinkle, 1969 Suborden ICHTHYODECTOIDEI Romer, 1966

Familia ICHTHYODECTIDAE Crook, 1892, sensu Blanco-Piñón \& Alvarado-Ortega, 2007

Xiphactinus Leidy, 1870

Especie tipo. Xiphactinus audax Leidy, 1870.

\section{Xiphactinus $\mathrm{sp}$.}

(Figuras 3-5)

Material referido. MT-G-070, ejemplar que conserva un pequeña parte la región nasal del cráneo, la parte anterior de las mandíbulas y vértebras abdominales de un solo ejemplar. Procedencia estratigráfica. Calizas del Cenomaniano del Miembro La Aguada, Formación La Luna. Cantera de la compañía "Cementos Andinos", a 17 km al noreste de Monay, Municipio Candelaria, Estado Trujillo, Venezuela.

Descripción. Proporciones generales: MT-G-070 conserva los fragmentos óseos de las mandíbulas, la región ethmoidea, $\mathrm{y}$ vértebras abdominales en tercera dimensión. Este conjunto óseo tiene una longitud cercana a los $16 \mathrm{~cm}$ y una anchura de $6 \mathrm{~cm}$. De acuerdo a la diagnosis del género Xiphactinus proporcionada por Bardack (1965, p. 37), la longitud de la 


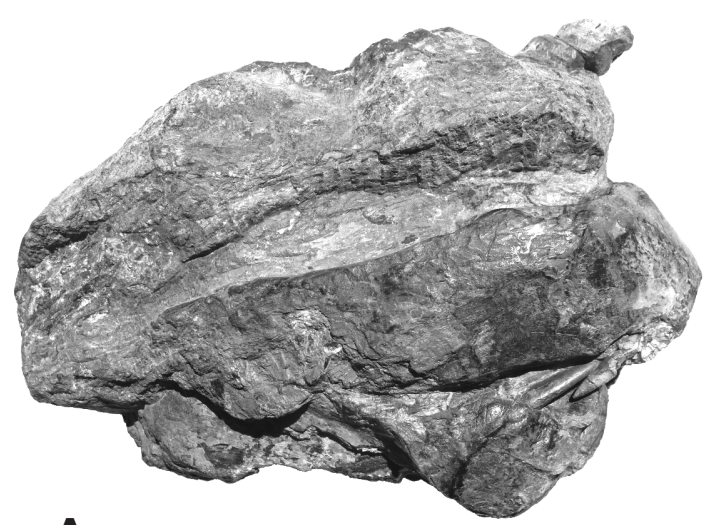

A

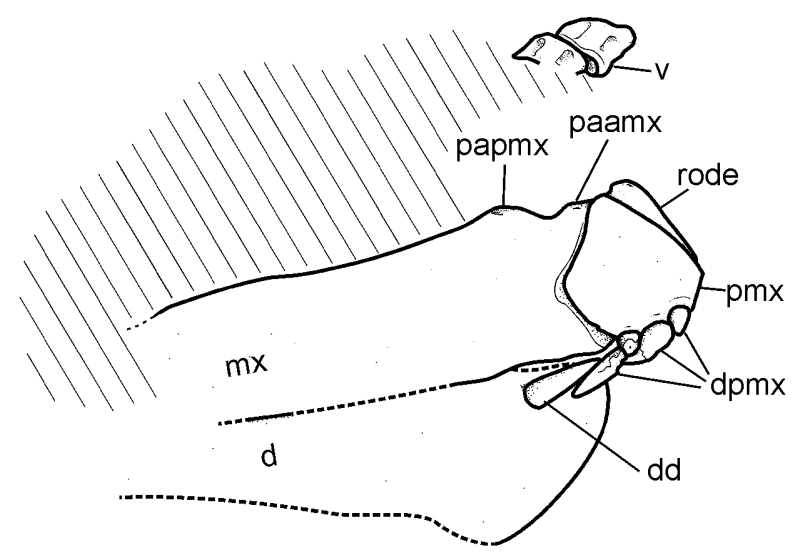

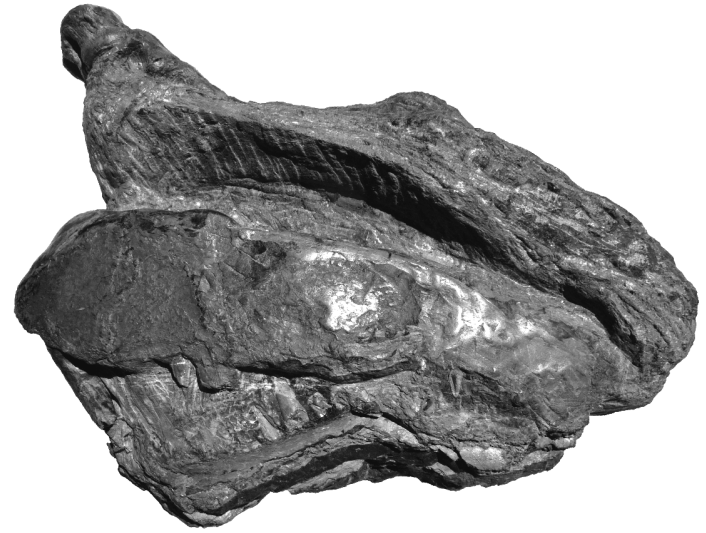

B

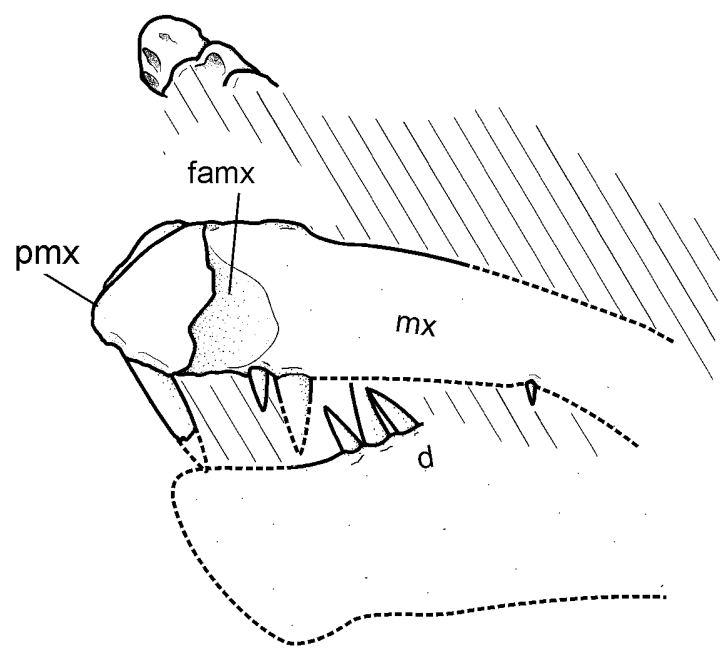

Figura 3. Fotografía y reconstrucción del ejemplar MT-G-070, Xiphactinus sp. de la Formación La Luna, Venezuela, en vista lateral derecha (A) e izquierda (B). Abreviaturas: d, dentario; dd, dientes del dentario; dpmx, dientes premaxilares; famx, fosa articular en el maxilar; $\mathbf{m x}$, maxilar; paamx, proceso articular anterior del palatino; papmx, proceso articular palatino del maxilar; pmx, premaxilar; rode, rostrodermethmoideo; $\mathbf{v}$, vértebras. Las líneas punteadas representan el tamaño aproximado de algunos huesos. Las líneas transversales representan la superficie de la roca portadora de este ejemplar. Escala $=5 \mathrm{~cm}$.

Figure 3. Picture and reconstruction of specimen MT-G-070, Xiphactinus sp. from La Luna Formation, Venezuela, in right (A) and left (B) lateral views. Abbreviations: $\mathbf{d}$, dentary; $\mathbf{d d}$, dentary teeth; $\mathbf{d p m x}$, premaxilla teeth; famx, articular fossa in the maxillary; $\mathbf{m x}$, maxilla; paamx, previous palatine articular process; papmx, palatine articular process of the maxillary; pmx, premaxilla; rode, rostrodermethmoid; $\mathbf{v}$, vertebrae. Dotted lines represent the approximate size of some bones. Transverse lines represent the carrier rock surface of this specimen. Scale bar $=5 \mathrm{~cm}$.

cabeza es contenida entre 5.5 y 6 veces en la longitud estándar. Por otro lado, a partir del ejemplar AMNH 1673, que cuenta con la cabeza completa, y de las figuras proporcionadas por Bardack (1965, fig. 9, pl. 1, fig. A), puede observarse que la longitud del premaxilar y el maxilar articulados, es ligeramente menor que la mitad de la longitud de la cabeza (incluyendo los huesos de la serie opercular). Con base en estos datos es posible determinar que en Xiphactinus la longitud del maxilar es contenida cerca de 11-12 veces en su longitud estándar. El premaxilar y maxilar izquierdos de MT-G-070 se conservan articulados y aun cuando el extremo posterior del maxilar está incompleto, estos huesos en conjunto miden al menos 11.5 $\mathrm{cm}$ a lo largo de su eje longitudinal (Figura 3); por lo tanto, la longitud estándar de MT-G-070 debió ser mayor a $1.50 \mathrm{~m}$ y tal vez, si se considera que cerca de la mitad del maxilar está conservada, éste no fue mayor de $3 \mathrm{~m}$.

Cráneo: el único hueso del cráneo conservado en MT-G070 está colocado por arriba de la región terminal anterior de la mandíbula superior (Figuras 3,4). Este es un hueso liso y aparentemente laminar, sus límites no están conservados por lo que no es posible definir su forma y sus dimensiones. Por su posición, es posible que se trate de restos del rostrodermethmoideo (ethmoideo en Bardack, 1965).

Mandíbula superior: Restos de ambas ramas de la mandíbula superior están conservados, incluyendo los dos premaxilares completos y sólo cerca de la mitad anterior de ambos maxilares (Figuras 3,4). Los premaxilares son huesos robustos de aspecto ovoide, ligeramente más altos que largos y con el borde anterior casi recto. Cada premaxilar es tan alto como su correspondiente maxilar, al cual se sobrepone y está firmemente articulado a través de la superficie articular o fosa articular del maxilar (famx en Figura 3) que forma una cavidad poco profunda en el extremo anterior externo del maxilar. En ambos premaxilares hay tres alvéolos ordenados formando una línea. A juzgar por los alveólos donde no hay dientes, éstos son cavidades profundas. Los dientes 


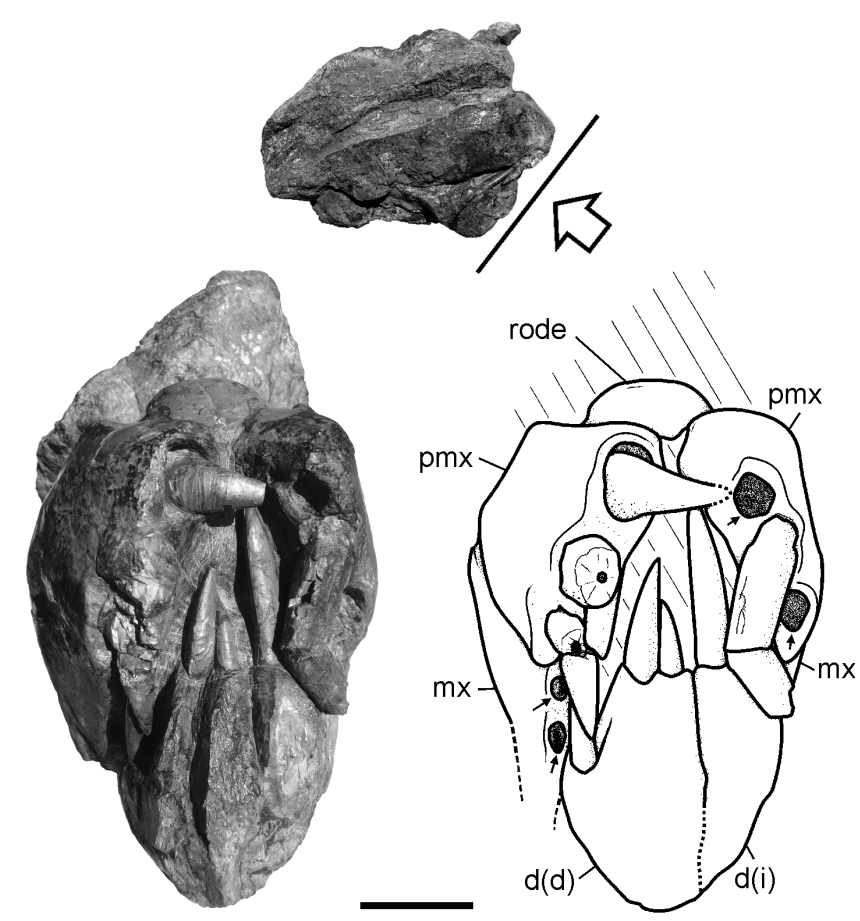

Figura 4. Fotografía y reconstrucción del ejemplar MT-G-070, Xiphactinus sp. de la Formación La Luna, Venezuela, en vista anteriorinferior. Arriba, la línea colocada en el extremo anterior inferior del ejemplar mostrado abajo. Abreviaturas: d, dentario; mx, maxilar; pmx, premaxilar; rode, rostrodermethmoideo. Las flechas indican las cavidades para los dientes o alvéolos vacios en los huesos maxilar y premaxilar. Escalas $=2 \mathrm{~cm}$.

Figure 4. Picture and reconstruction of specimen MT-G-070, Xiphactinus sp. from La Luna Formation, Venezuela, in anterior-lower view. Above, the line in the anterior-lower part from the specimen shown below. Abbreviations: d, dentary; $\mathbf{m x}$, maxilla; pmx, premaxilla; rode, rostrodermethmoid._Arrows indicate cavities or alveoli for teeth in maxillary and premaxilla bones. Scale bars $=2 \mathrm{~cm}$.

conservados son caniniformes, lisos, robustos y cónicos. En el premaxilar derecho todos los alvéolos están ocupados por dientes (Figura 4); la altura de la corona del diente anterior es de al menos $2 \mathrm{~cm}$ (no conserva su punta); por otro lado, el diente central sólo conserva la raíz y la base de la corona dentro del alvéolo mostrando que en su corte transversal estos dientes son circulares y presentan un pequeño hueco central; el diente posterior también está roto desde su base pero casi toda la corona de al menos $2.2 \mathrm{~cm}$ de altura está inclinada hacia atrás. En el premaxilar izquierdo el alvéolo central está ocupado por un diente que está fracturado en la parte media y cuya punta está rota; por eso, su altura sólo puede ser señalada como mayor a $5.5 \mathrm{~cm}$. Ambos maxilares están conservados sólo parcialmente; aunque el extremo posterior de estos huesos no está conservado, su extremo anterior tiene un aspecto rectangular, casi recto y uniformemente profundo (Figura 3). La longitud de estos huesos es de al menos 15 $\mathrm{cm}$ a lo largo de su eje longitudinal y su altura es próxima a los $5 \mathrm{~cm}$. En el maxilar derecho es clara la presencia de un par de procesos articulares, el proceso palatino (éste permite la articulación del maxilar con el palatino) y el proceso anterior (éste articula el maxilar con una faceta formada entre el ethmopalatino y el vómer (Patterson \& Rosen, 1977, fig.
6), poco elevados y de aspecto redondeado, ubicados sobre el borde dorsal del extremo anterior. Los premaxilares están sobrepuestos y articulados firmemente a la superficie articular de sus respectivos maxilares, en el maxilar dicha articulación se lleva a cabo a través de una fosa articular del maxilar (famx) que es una cavidad poco profunda cuya parte posterior es más evidente en el maxilar izquierdo. Aunque el número de alvéolos en cada maxilar no es claro, en el maxilar derecho es posible observar al menos dos alvéolos profundos (Figura 4) y el maxilar izquierdo se conservan tres dientes cónicos, robustos, lisos y de talla irregular que relativamente son los más pequeños observados en MT-G-070 (Figura 3). El primero de estos dientes, el colocado cerca del extremo anterior de este hueso es menor de $2 \mathrm{~cm}$; el diente conservado en la parte media de este hueso, que sólo conserva parte basal de su corona, pudo tener una altura entre los 3 y $4 \mathrm{~cm}$; el último de los dientes maxilares conservado, que está colocado por detrás de los ya descritos, es ligeramente mayor a $1 \mathrm{~cm}$.

Mandíbula inferior: desafortunadamente la mandíbula inferior está representada sólo por fragmentos de la región alveolar de ambos dentarios. El borde alveolar de estos fragmentos es aparentemente recto y muestra dientes caniniformes, lisos robustos, cónicos, de talla irregular (Figura 3). En la cara anterior de este fósil es posible observar tres dientes del dentario derecho y uno del dentario izquierdo conservados por debajo de los premaxilares (Figura 4). La altura de los tres dientes conservados en el dentario derecho son en orden anteroposterior de 2, $2.5 \mathrm{y}$ al menos $3.5 \mathrm{~cm}$, respectivamente. El primero de dichos dientes, el colocado posteriormente y el único que puede ser observado lateralmente (Figura 3), parece estar implantado sobre la cara lateral externa del dentario; sin embargo, dicho diente está inclinado hacia adelante y arriba provocando este efecto visual. En el dentario izquierdo hay cuatro dientes conservados, tres de ellos pueden ser observados lateralmente (Figura 3) y otro anteriormente (Figura 4). El ápice del diente intermedio observable en vista lateral está cubierto por el maxilar correspondiente. La altura de los dientes restantes en orden anteroposterior es de al menos 4,3 y 3.3 $\mathrm{cm}$, respectivamente. Es necesario señalar que los dientes conservados entre los maxilares al parecer tienen una sección transversal ovoide y un borde cortante o carinae anterior (Figura 4). Dado que ninguno de los otros dientes presenta estos rasgos, es posible concluir que dicha deformación es un artefacto de conservación y sugerir que en algún momento de la diagénesis, éstos fueron deformados por compresión.

Vértebras: en MT-G-070 hay cuatro vértebras articuladas contenidas en la misma matriz calcárea sobre la que descansan los restos óseos antes descritos. Dichas vértebras son cilíndricas, más altas que largas, con la cara intervertebral casi circular y presentan un diámetro de $3.5 \mathrm{y}$ en vista lateral su longitud es de $1.5 \mathrm{~cm}$. y su altura alcanza los $2 \mathrm{~cm}$. En la última de estas vértebras es posible observar una concavidad profunda que en el centro muestra una pequeña perforación o canal del notocordo. En el borde dorsal de estas vértebras hay dos facetas longitudinales profundas en donde se fija el arco neural (fan en Figura 5). En la cara lateral externa de estas 


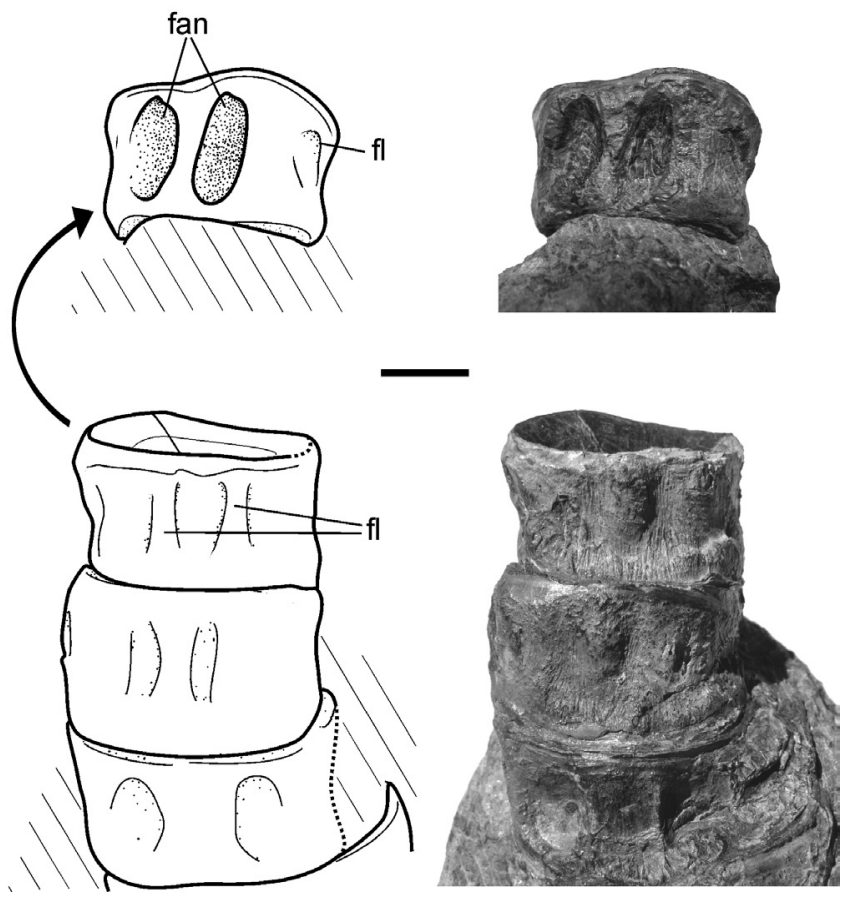

Figura 5. Fotografía y reconstrucción de las vértebras conservadas en el ejemplar MT-G-070, Xiphactinus sp. de la Formación La Luna, Venezuela. Abajo, cara lateral de las cuatro vértebras conservadas mostrando el par de fosas laterales (fl) en su cara lateral externa. Arriba, vista dorsal de la vértebra colocada en el ápice de la secuencia de vértebras conservadas donde observan las facetas para la articulación del arco neural (fan). Escala $=1 \mathrm{~cm}$.

Figure 5. Picture and reconstruction of the vertebrae from specimen MT-G-070, Xiphactinus sp. from La Luna Formation, Venezuela. Below, lateral view of the four vertebrae showing the pair of lateral cavities (fl) in its lateral external view. Above, dorsal view from the vertebrae putted in the beginning of the vertebrae sequence where the facets for the articulation of the neural arch are observed (fan). Scale bar $=1 \mathrm{~cm}$.

vértebras también existe un par de cavidades longitudinales, pero estas son menos profundas. No hay evidencia de superficies articulares para la fijación de los arcos hemales, lo que sugiere que se trata de vértebras abdominales.

\section{DISCUSIÓN}

Los rasgos conservados en MT-G-070 son tan pocos que no permiten determinar de manera contundente su identidad taxonómica a nivel específico; sin embargo, aquellos ya descritos en la sección anterior permiten identificarlo como un ejemplar perteneciente al género Xiphactinus. Su posición dentro del suborden Ichthyodectoidei está bien fundamentada en la sección dentada del maxilar que es recta y uniformemente profunda, rasgo señalado por (AlvaradoOrtega, 2005, p.180-181) como diagnóstico de este suborden. Al mismo tiempo, éste puede ser reconocido como miembro de la familia Ichthyodectidae debido a que presenta dientes premaxilares hipertrofiados, previamente señalados por Blanco-Piñón \& Alvarado-Ortega (2007) como uno de los rasgos diagnósticos de la familia. Finalmente, la presencia de dientes de talla irregular en los dentarios y maxilares es un rasgo diagnóstico exclusivo de Xiphactinus (e.g. Bardack,
1965); este patrón morfológico presente en el ejemplar MTG-070, permite referir el mismo dicho género.

Aunque Blanco-Piñón \& Alvarado-Ortega (2007) reconocieron que la familia Ichthyodectidae se caracteriza por presentar dientes premaxilares alargados; la condición en el tamaño y número de dientes premaxilares presentes en Xiphactinus ha sido señalada como un hipertrofia por Schwimmer et al. (1997). De acuerdo a estos últimos autores, sólo en Xiphactinus han sido registrados diente premaxilares de hasta $8 \mathrm{~cm}$ longitud. Aunque incompleto, en MT-G070 el único diente conservado en el premaxilar izquierdo puede ser descrito como hipertrofiado (su longitud es de al menos $5.5 \mathrm{~cm}$ ), sobre todo si se considera que este fósil venezolano representa un ejemplar pequeño (de longitud estándar cercana a $1.5 \mathrm{~m}$ ) en comparación con Xiphactinus encontrados en Norteamérica, donde estos enormes dientes han sido registrados con longitudes cuatro veces mayores (5-6 m). La hipertrofia de los dientes premaxilares en los Ichthyodectidae también involucra el número de alveólos donde están colocados. De acuerdo con Bardack (1965), Xiphactinus se caracteriza por presentar el menor número de alvéolos premaxilares, los cuales en promedio son 2.6 alvéolos en cada premaxilar (rango entre 2 y 5 ). Por el contrario, otros miembros de la familia (sensu Blanco-Piñón \& AlvaradoOrtega, 2007), Ichthyodectes Cope, 1870 y Vallecillichthys Blanco-Piñón \& Cavin, 2003, en donde este fenómeno de hipertrofia dentaria es más elocuente, en promedio hay 7 (entre 3 y 12) y al menos 6 alvéolos premaxilares respectivamente (Bardack, 1965; Blanco-Piñón \& Alvarado-Ortega, 2007). A pesar de que el número de alveólos premaxilares del ejemplar MT-G-070 es próximo al del promedio de Xiphactinus y es igual al límite inferior de Ichthyodectes, difiere de este último ya que este género no presenta dientes de talla irregular en el maxilar como en Xiphactinus.

Actualmente el género Xiphactinus incluye cuatro especies nominales, la especie tipo excepción de $X$ audax Leidy, 1870 y las especies $X$. vetus (Leidy, 1870), X. gaultinus Newton, 1877 y X. mantelli Newton, 1877. De acuerdo con Bardack (1965) y Schwimmer et al. (1997), con excepción de X. audax y $X$. vetus, los rasgos distintivos de las otras especies de este género son escasos y sólo han sido señalados tentativamente como posibles rasgos diagnósticos. Xiphactinus mantelli y $X$. gaultinus posiblemente son diferentes de $X$. audax porque en estas especies "el proceso o cóndilo palatino del maxilar es un poco elevado" y entre estas especies $X$. gaultinus presenta "dientes maxilares de talla relativamente uniforme"; sin embargo, ambas especies son consideradas sólo "provisionalmente distintas de $X$. audax principalmente por su distribución geográfica" (Bardack, 1965, p. 54). De acuerdo con Schwimmer et al. (1997), en X. vetus, conocido solo por dientes y vértebras, los dientes son alargados, ligeramente recurvados, moderadamente comprimidos lateralmente (en corte transversal los dientes son ovalados o poligonales), con un fuerte desarrollo de carinae o borde cortante a lo largo de todo su margen anterior y posterior. Como se señaló antes, sólo los dientes dentarios observados entre las premaxilas del ejemplar MT-G-070 no son cónicos 
y su corte transversal debe ser ovalado; sin embargo, en este trabajo se ha considerado que estos dientes fueron deformados por una fuerte compresión en algún momento de la diagénesis. Al no encontrar claras evidencias de carinae en los dientes de MT-G-070 es posible descartar la posibilidad de que este pertenezca a la especie $X$. vetus.

Finalmente, por el momento el aspecto "elevado" del proceso palatino en el maxilar, considerado por Bardack (1965) para sugerir que Xiphactinus audax es diferente de otras especies del género, es un rasgo que no ha sido estudiado detalladamente. Ante la falta de certidumbre sobre el significado taxonómico de esta característica es imposible diferenciar a $X$. audax (con este proceso aparentemente más elevado) de $X$. matelli y $X$. gaultinus (donde este proceso es menos elevado) y por lo tanto no se puede reconocer la identidad específica del ejemplar MT-G-070, que bajo los argumentos señalados es considerado como Xiphactinus sp.

\section{IMPLICACIONES PALEOBIOGEOGRÁFICAS}

Antes de este trabajo, el patrón en la distribución paleobiogeográfica de las cuatro especies nominales del género Xiphactinus involucraba localidades cretácicas de América del Norte y Europa (Bardack, 1965; Schwimmer et al., 1997). X. audax es la especie mejor documentada hasta ahora gracias a un amplio registro de ejemplares completos y fragmentarios colectados en yacimientos del Cretácico (Albiano-Campaniano) de Estados Unidos de Norteamérica (en Kansas, Nebraska, Texas, Alabama y Arkansas) y Canadá (Manitoba). X. gaultinus está representado por fósiles escasos y fragmentados del Cretácico "medio" (Albiano-Cenomaniano) colectados en Folkstone y Kent, Inglaterra. X. mantelli está representado por fósiles escasos y fragmentarios colectados en sedimentos del Cretácico Superior (Cenomaniano-Santoniano) de Inglaterra, Polonia, República Checa, Bélgica y Francia. Finalmente, X. vetus es una especie re-descrita por Schwimmer et al. (1997) que está representada por fósiles colectados en yacimientos del Cretácico Superior (Campaniano-Maastrichtiano) en Estados Unidos de Norteamérica (Mississipi, Alabama, Georgia, North Carolina, Delawere y New Jersey). Por lo tanto, la distribución paleobiogeográfica de Xiphactinus hasta ahora conocida incluye yacimientos marinos depositados dentro del dominio del supercontinente Laurasia, ubicados dentro del Mar Interior de Norteamérica y el margen septentrional del Mar de Tetis, en los que ahora es Europa. Esto hace del ejemplar MT-G-070 el primer registro confiable del género Xiphactinus fuera de Europa América del Norte, y el primero recuperado en yacimientos depositados dentro del dominio del Supercontinente Gondwana.

Otros reportes, ya desestimados, del género Xiphactinus dentro del dominio de Gondwana incluye a dos especies australianas originalmente colocadas en el género Portheus Cope, 1872, que actualmente es considerado sinónimo de Xiphactinus (Bardack, 1965, p. 54). La especie X. australis (Woodward, 1894) está descrita a partir de un fragmento del hocico y algunas vértebras colectados en sedimentos marinos del Cretácico Superior de la Formación Rolling Downs, en las cercanías de Hughenden, Queensland, que actualmente están incluidos en el material tipo de otra especie ichthyodectiforme llamada Cooyoo Lee \& Bartholomai, 1987. Otra especie, Portheus dunedinensis Chapman, 1933, fue encontrada en los estratos paleógenos de glauconita en Abbotsford, cerca de Dunedin, Nueva Zelanda; una revisión posterior permitió identificarlo como un pez pachyrhizodontido (Bardack, 1962, 1965).

En México, Maldonado-Koerdell (1956) reportó la presencia de Xiphactinus en los sedimentos marinos de Xilitla (Formación Agua Nueva), en Estado de San Luis de Potosí; sin embargo, Alvarado-Ortega (2005) desestimó la ocurrencia de Xiphactinus en México porque los restos fósiles de Ichthyodectiformes de gran tamaño hasta ahora encontrados en el país, en ésta y otras localidades al norte, no muestran los rasgos diagnósticos de dicho género.

Aún cuando los estudios sobre peces óseos del Cretácico de Venezuela son escasos (Odreman \& Medina, 1984; Moody \& Maisey, 1994; Sánchez-Villagra et al., 2008; Carrillo, 2009), la presencia de peces Ichthyodectiformes en este país incluye el reporte de ejemplares conferidos a Ichthyodectes (Moody \& Maisey, 1994) y Cladocyclus Agassiz, 1841 (Aguilera \& Lundberg, 2009, Aguilera, 2010), recolectados en las calizas del Aptiano de la Formación Apón, en la Sierra de Perijá, al Oeste de Venezuela. Cladocyclus es un Ichthyodectiforme gondwánico ampliamente documentado en las formaciones Crato y Santana también presente en las cuencas de Araripe, Parnaíba y Sergipe-Alagoas (Brito \& Yabumoto, 2011) recientemente encontrado en los yacimientos marinos del Cenomaniano Tardío-Turoniano Temprano de Gara Sbaa, en Marruecos (Martill et al., 2011). Ichthyodectes, también miembro de la familia Ichthyodectidae, tiene una distribución temporal y geográfica semejante pero ligeramente más amplia a la ya señalada para Xiphactinus, sus restos han sido colectados en depósitos marinos del Cretácico (Albiano-Maastrichtiano) del Mar Interior de Norte América, yacimientos marinos del norte del Mar de Tetis en Europa, y además en Marruecos (Bardack, 1965, p. 54-62). Desafortunadamente, los restos fósiles atribuidos a Ichthyodectes y Cladocyclus encontrados en Venezuela sólo han sido estudiados superficialmente y aún se requiere la confirmación de dichas determinaciones taxonómicas.

El hallazgo de Xiphactinus en América del Sur se suma al reconocimiento de otros géneros ichthyodectiformes cretácicos típicos del de América del Norte y Europa, que en realidad tuvieron una distribución mucho más amplia que involucra los dominios de Laurasia y Gondwana. En este caso se incluye la presencia ya señalada de Ichthyodectes en América del Sur y los restos de Saurocephalus encontrados en las fosforitas maastrichtianas del Forno de Cal, Formación Gramame, en las cercanías Olinda, Estado de Pernambuco, Brasil (Santos \& Figueiredo, 1987).

\section{CONCLUSIONES}

Algunos elementos característicos de los Ichthyodectiformes, 
como los centros vertebrales, con sus dos pares de facetas dorsales para la articulación de los arcos neurales, un premaxilar corto portados de dientes hipertrofiados y poco abundantes, en donde los bordes dorsal y ventral de la sección dentada son rectos y paralelos, y la presencia de dientes de talla irregular en el maxilar, premaxilar y dentario, permiten concluir que el ejemplar MT-G-070, pertenece al género Xiphactinus, dentro del orden Ichthyodectiformes. Aunque la morfología dental de este ejemplar permite diferenciarlo claramente de $X$. vetus, no existe información disponible suficiente para establecer si este ejemplar pertenece o no a alguna de las otras especies nominales del género.

El presente registro de Xiphactinus representa el primer hallazgo de peces ichthyodectiformes en la Formación La Luna y el primero del Cretácico superior en Venezuela. De la misma manera, representa el primer reporte del género en toda América del Sur y el primero dentro de lo que fue Gondwana.

\section{AGRADECIMIENTOS}

A M. Everhart (Sternberg Museum of Natural History de Kansas) por su valiosa colaboración y recomendaciones. Al A. Cione (Universidad Nacional de la Plata) y al S. Bogan (Fundación de Historia Natural "Félix de Azara") por sus valiosos aportes para la elaboración de este trabajo.

\section{REFERENCIAS}

Agassiz, L. 1833-1844. Recherches sur les poissons fossiles: 5 vols. With atlas (Neuchâtel).

Aguilera, O. 2010. Peces Fósiles del Caribe de Venezuela. USA, Gorham Printing, Centralia, $258 \mathrm{p}$.

Aguilera, O. \& Lundberg, J. 2009. Venezuelan Caribbean and orinocoan Neogene Fish. In: M. Sánchez-Villagra; O. Aguilera \& F. Carlini (eds.) Urumaco and Venezuelan paleontology - The fossil record of the northern neotropics, Indiana Press University, p. $129-152$.

Alvarado-Ortega, J. 2005. Sistemática de los peces Ichthyodectiformes de la cantera Tlayúa, Puebla, México. Posgrado en Ciencias Biológicas, Universidad Nacional Autónoma de México, Tesis de Doctorado, 302 p.

Arratia, G.; Scasso, R.A. \& Kiessling, W. 2004. Late Jurassic fishes from Longing Gap, Antarctic Peninsula. Journal of Vertebrate Paleontology, 24:41-51. doi:10.1671/1952-4

Bardack, D. 1962. Taxonomic status and geologic position of the Cretaceous fish Ichthyodectes marathonensis. Australian Journal of Science, 24:387.

Bardack, D. 1965. Anatomy and evolution of chirocentrid fishes. The University of Kansas Paleontological Contributions, Vertebrata, 10:1-88.

Bardack, D. \& Sprinkle, G. 1969. Morphology and relationships of saurocephalid fishes. Fieldiana Geology, 16:297-340.

Blanco-Piñón, A. \& Alvarado-Ortega, J. 2007. Review of Vallecillichthys multivertebratum (Teleostei: Ichthyodectiformes), a Late Cretaceous (Early Turonian) "Bulldog fish" from Northeastern Mexico. Revista Mexicana de Ciencias Geológicas, 24:450-466.

Blanco-Piñón, A. \& Cavin, L. 2003. New Teleostei from the Agua Nueva Formation (Turonian), Vallecillo (NE Mexico). Comptes Rendus Palevol, 2:299-306. doi:10.1016/S1631-0683(03)00064-2

Brito, P. \& Yabumoto, Y. 2011. An updated review of fish faunas from Crato and Santana formations in Brazil, a close relationship to the Tethys fauna. Bulletin Kitakyushu Museum of Natural History and Human History, 9:107-136.

Carrillo, J. 2009. Presencia del género Ptychodus (Elasmobranchii: Ptychodontiade) en el Cretácico superior de los Andes de Trujillo Venezuela. Geominas, 37:207-210.

Chapman, F. 1933. Description of fossil fish from New Zeland. Transactions of the New Zeland Institute, 64:117-121.

Cope, E.D. 1870. On the Saurodontidae. American Philosophical Society, Proceedings, 11:529-538.

Cope, E.D. 1872. On the geology and paleontology of the Cretaceous strata of Kansas: United States Geological Survey of Montana, 5th Ann. Rept. (F. V. Hayden), p. 318-349.

Crook, A.R. 1892. Über einige fossile Knochenfi sche aus der mittleren Kreide von Kansas. Palaeontographica, 39:107-124.

Davis, C.L. 1997. Paleoceanographic influences on accumulation sedimentologic of organic matter and trace metals in Cretaceous black shale and carbonate, western Maracaibo Basin, Venezuela. Indiana University, Ph.D. thesis, 348 p.

De Romero, L.M.; Truskowski, I.M.; Brawler, T.J.; Bergen, J.A.; Odreman, O.; Zachos, J.C. \& Galea-Alvarez, F.A. 2003. An integrated calcareous microfossil biostratigraphic and carbonisotope stratigraphic framework for the La Luna Formation, western Venezuela. Palaios, 18:349-366. doi:10.1669/08831351(2003)018<0349:AICMBA>2.0.CO;2

Everhart, M.J.; Hageman, S.A. \& Hoffman, B.L. 2010. Another Sternberg "fish-within-a-fish" discovery: first report of Ichthyodectes ctenodon (Teleostei; Ichthyodectiformes) with stomach contents. Transactions of the Kansas Academy of Science, 113:197-205. doi:10.1660/062.113.0306

Garner, A.H. 1926. Suggested nomenclatural and correlation on geological formations in Venezuela. American Institute of Mining and Metallurgy Engineers, Trasactions, Petroleum Development and Technology in 1925, p. 677-684.

Hedberg, H. \& Sass, L. 1937. Synopsis of the geologic formations of the western part of the Maracaibo Basin, Venezuela. Boletin de Geología y Mineria, 2-4:71-112.

Lees, T. \& Bartholomai, A. 1987. Study of a Lower Cretaceous actinopterygian (Class Pisces) Cooyoo australis from Queensland, Australia. Memoirs of the Queensland Museum, 25:177-192.

Leidy, J. 1870. Remarks on ichthyodorulites and on certain fossil mammalians. Proceedings of the American Philosophical Society, 22:12-13.

Lorente, M. A.; Duran, I. \& Ruiz, M. 1996. Late Cretaceous in western Venezuela, a new biostratigraphical approach. In: AAPG/ SVG INTERNATIONAL CONGRESS AND EXHIBITION, 2, 1996. Abstracts, Caracas, AAPG, 80:1309-1310.

Maldonado-Koerdell, M. 1956. Peces fósiles de México III. Nota preliminar sobre peces del Turoniano superior de Xilitla, San Luis Potosí, México. Ciencia, 16:31-36.

Martill, D.B.; Ibrahim, N.; Brito, P.M.; Baidder, L.; Zouhri, S.; Loveridge, R.; Naish, D. \& Hing, R. 2011. A new plattenkalk Konservat Lagerstätte in the Upper Cretaceous of Gara Sbaa, south-eastern Morocco. Cretaceous Research, 32:433-446. doi:10.1016/j.cretres.2011.01.005

Moody, J. \& Maisey, J. 1994. New cretaceous marine vertebrate assemblages from north-western Venezuela and their significance. Journal of Vertebrate Paleontology, 14:1-8.

Newton, E.T. 1877. On the remains of Hypsodon, Portheus and Ichthyodectes from British Cretaceous strata, with descriptions of a new species. Quarterly Journal of the Geological Society of London, 33:505-529. 
Odreman, O. \& Medina, C. 1984. Vertebrados fósiles de Venezuela, secuencia, relaciones con otros países de America del Sur. Ministerio de Energía y Minas, Cuadernos de Geología 1:60-86.

Patterson, C. \& Rosen, D.E. 1977. Review of ichthyodectiform and other Mesozoic teleost fishes and the theory and practice of classifying fossils. Bulletin of the American Museum of Natural History, 158:81-172.

Renz, O. 1959. Estratigrafía del Cretáceo en Venezuela occidental. Boletín de Geología, 5:3-48.

Romer, A.S. 1966. Vertebrate Paleontology. Chicago, University Press, 468 p.

Sánchez-Villagra, M.R.; Brinkmann, W. \& Lózsan, R. 2008. The Palaeozoic and Mesozoic vertebrate record of Venezuelaan overview, summary of previous discoveries and report of a mosasaur from the La Luna Formation (Cretaceous). Paläontologische Zeitschrift, 82:113-124. doi:10.1007/ BF02988403

Santos, R.S. \& Figueiredo, F. J. 1987. Sobre um Saurocephalidae da Formação Gramame (Camada de Fosfato), Estado de Pernambuco. In: CONGRESSO BRASILEIRO DE PALEONTOLOGIA, 10, 1987. Resumos, Rio de Janeiro, UFRJ, p. 4.

Schaeffer, B. \& Patterson, C. 1984. Jurassic fishes from the western United States, with comments on Jurassic fish distributions.
American Museum Novitates, 2796:1-86.

Schwimmer, D.; Stewart, J.D. \& Williams, G.D. 1997. Xiphactinus vetus and the distribution of Xiphactinus species in the eastern United States. Journal of Vertebrate Paleontology, 17:610-615.

Tanimoto, M. \& Kinkyo, T. 2001. Gillicus skeleton from the Upper Cretaceous Izumi Group (Lower Maastrichtian) of Yura-Cho, Sumoto City, Hyogo Prefecture, Southwest Japan the first find of a fossil of the family Ichthyodectidae in Japan. Chigakukenkyu, 50:41-43.

Taverne, L. 2010. Les Ichthyodectidae (Teleostei, Ichthyodectiformes) des schistes bitumineux de l'Aptien (Crétacé inférieur) de Guinée Équatoriale et du Gabon. Bulletin de L'Institut Royal des Sciences Naturelles de Belgique, Sciences de la Terre, 80:115-143.

Woodward, A. 1894. On some fish-remains of the genera Portheus and Cladocyclus from the Rolling Downs Formation (lower Cretaceous) of Queensland. Annals and Magazine of Natural History, 6:444-447.

Zapata, E.; Padro, V.; Madrid, I.; Kertznus, V.; Truskowski, I. \& Lorente, M.A. 2003. Biostratigraphic, sedimentologic, and chemostratigraphic study of the La Luna Formation (Late Turonian-Campanian) in the San Miguel and Las Hernández sections, western Venezuela. Palaios, 18:367-377. doi:10.1669/0883-1351(2003)018<0367:BSACSO > 2.0.CO;2

Received in September, 2011; accepted in September, 2012. 